\title{
Supraventricular Tachycardia Originating From the Posterior Atrioventricular Node in the Univentricular Heart With Single Atrium
}

\author{
Kohsuke AJIKI, ${ }^{1} \mathrm{MD}$, Noriyuki HAYAMI, ${ }^{1} \mathrm{MD}$, Yuji KASAOKA, ${ }^{1} \mathrm{MD}$, \\ Yasushi IMAI, ${ }^{1} \mathrm{MD}$, Katsuhito FuJIU, ${ }^{1} \mathrm{MD}$, and Yuji MuraKAwA, ${ }^{1} \mathrm{MD}$
}

\section{SUMMARY}

Supraventricular tachycardia (SVT) was observed in a 13-year-old male patient with complex clinical features that included univentricular heart with single atrium, pulmonary atresia, and polysplenia syndrome. During electrophysiologic study, atrial burst stimuli reproducibly induced and terminated the SVT, while the occurrence of ventriculoatrial block did not interrupt the SVT. His bundle electrograms (HBEs) were recognized both in the anterior and posterior regions on the common atrioventricular (AV) valve annulus. The posterior His bundle activation was progressively delayed along with the shortening of atrial pacing cycle length until it finally lagged behind local ventricular activation. Thus, antegrade AV conduction was solely via the anterior AV node. In contrast, during the SVT, the earliest activation was observed in the posterior HBE. These observations suggested that the posterior AV node serves as an origin of the SVT and that two AV nodes were linked together possibly through a sling at the infra-Hisian level. Radiofrequency catheter ablation applied to the posterior HBE eliminated the SVT. (Int Heart J 2007; 48: 253-259)

Key words: Supraventricular tachycardia, Univentricular heart, Single atrium, Dual atrioventricular nodes, Radiofrequency catheter ablation

IN complex congenital heart diseases such as situs ambiguus and corrected transposition of the great arteries, the existence of dual atrioventricular (AV) nodes connected by a sling has been described. ${ }^{1-3)}$ Dual AV nodes are prone to cause inter-AV node reentrant tachycardia. ${ }^{4-6)}$ In this report, we present a case of univentricular heart whose SVT occurred via a mechanism other than inter-AV node reentry despite the presence of dual AV nodes.

\footnotetext{
From the ${ }^{1}$ Department of Cardiovascular Medicine, Graduate School of Medicine, The University of Tokyo, Tokyo, Japan. Address for correspondence: Kohsuke Ajiki, MD, The Department of Cardiovascular Medicine, The University of Tokyo, 7-3-1 Hongo, Bunkyo-ku, Tokyo 113-8655, Japan.

Received for publication October 10, 2006.

Revised and accepted February 5, 2007. 


\section{CASE Report}

A 13-year-old boy had been diagnosed at birth with single atrium, single ventricle (morphologically right ventricle to the right of rudimentary left ventricle), pulmonary atresia, and polysplenia syndrome. A Blalock-Taussig operation was performed when he was 1 year old. He had suffered from frequent episodes of paroxysmal SVT since the age of 5. Antiarrhythmic agents including digoxin and verapamil had failed to control his SVT. He was referred to our department for evaluation and treatment of the SVT.

After written informed consent was obtained, an electrophysiologic (EP) study was performed after all antiarrhythmic medications except digoxin had been discontinued for longer than 5 drug half-lives. A midazolam drip was used for sedation, with intermittent midazolam and pentazocine boluses as needed. Atrial burst stimuli reproducibly induced and terminated the clinical SVT at a pacing cycle length of 286 and $272 \mathrm{msec}$, respectively. The SVT cycle length

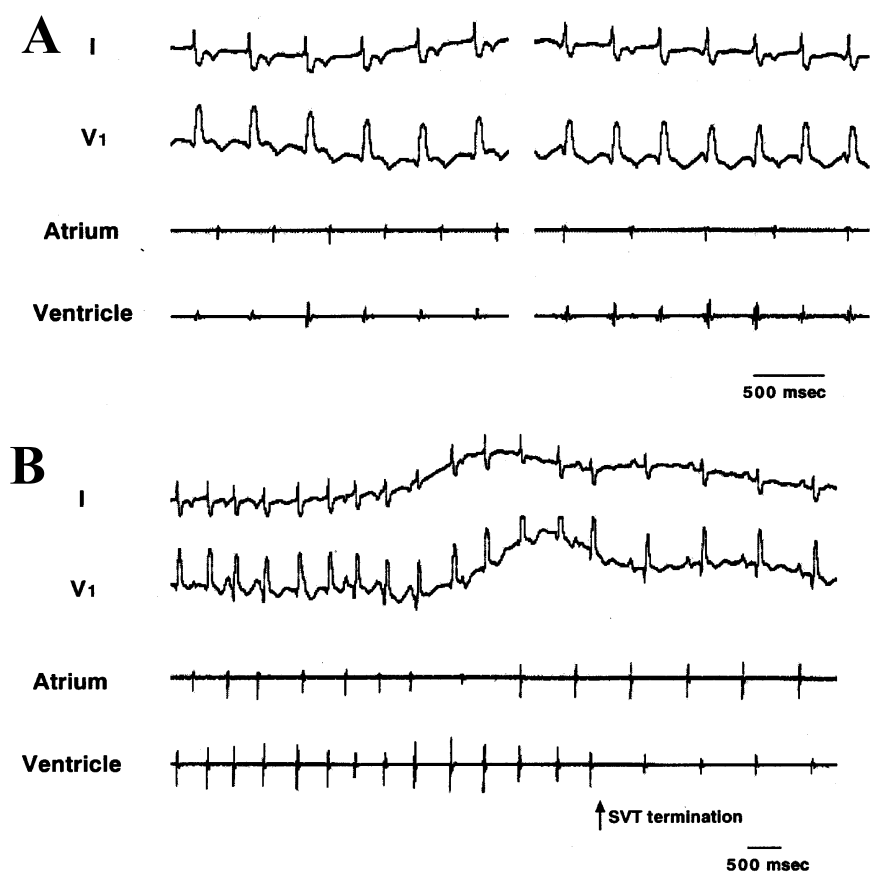

Figure 1. Body surface ECG leads I and V1, and intracardiac electrograms recorded in the atrium and the ventricle during the SVT. A: The SVT cycle length was $430 \mathrm{msec}$ in the left panel, and $390 \mathrm{msec}$ in the right panel. The SVT cycle length shorter than $400 \mathrm{msec}$ resulted in Wenckebach VA block without affecting the SVT cycle length. B: The bolus injection of ATP $(5 \mathrm{mg})$ terminated the SVT. Transient VA dissociation was observed immediately before the SVT termination. 
A

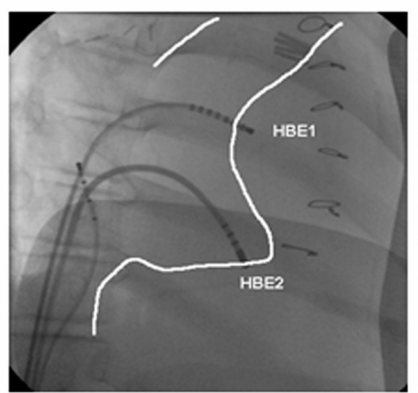

RAO

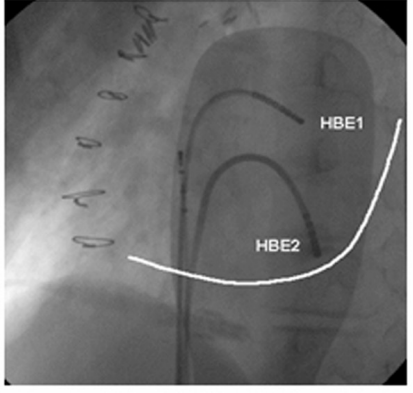

LAO

B

I
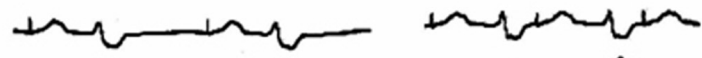

$\mathbf{V}_{1}$

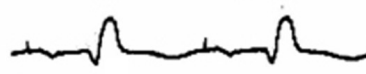

Atrium

HBE1
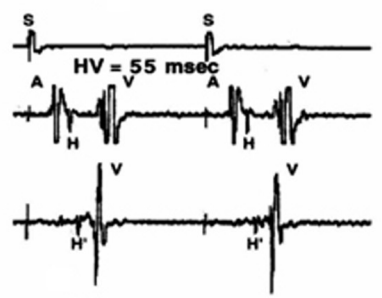

$H^{\prime}=25$ msec
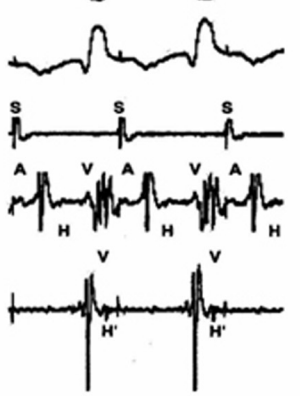

$H^{\prime}=135 \mathrm{msec}$

C

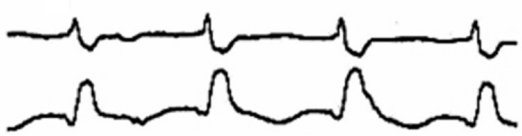

Atrium

HBE1

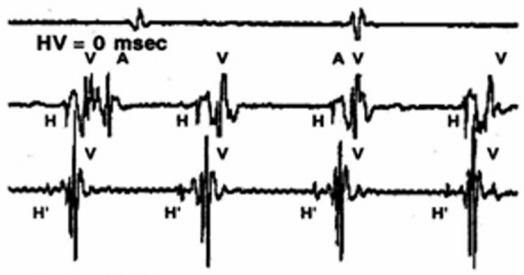

$H^{\prime} \mathbf{H}=25 \mathrm{msec}$

$500 \mathrm{msec}$

Figure 2. A: The left panel shows the right anterior oblique view, and the right panel, the left anterior oblique view. HBE1 and HBE2 represent the anterior and the posterior sites at which His bundle electrocardiograms were recorded on the common AV valve annulus, respectively. The white line shows the atrial contour which was preliminarily ascertained by atriography. B: Body surface ECG leads I and V1, and intracardiac electrograms recorded in the atrium, HBE1, and HBE2 during atrial pacing of $500 \mathrm{msec}$ (left panel) and $300 \mathrm{msec}$ (right panel). S, A, H, H', and V indicate pacing spike, atrial deflection, His bundle deflection in HBE1, His bundle deflection in HBE2, and ventricular deflection, respectively. C: Body surface ECG leads I and V1, and intracardiac electrograms recorded in the atrium, HBE1, and HBE2 during the SVT with a cycle length of $370 \mathrm{msec}$. The abbreviations are the same as in Figure 2B. 
varied between 430 and $345 \mathrm{msec}$. SVT cycle length below $400 \mathrm{msec}$ caused Wenckebach ventriculoatrial (VA) block but did not affect tachycardia cycle length (Figure 1A). Ventricular extrastimulation did not reset the SVT. The bolus injection of ATP (5 mg) terminated the SVT (Figure 1B).

Detailed mapping along the common AV valve annulus identified two His bundle electrograms (HBEs) in the anterior and posterior regions, which presumably correspond to the anterior and posterior AV nodes, respectively (Figure 2A). Marked progressive delay of the posterior His bundle activation was observed as the atrial pacing cycle length was shortened, and finally, the posterior His bundle activation was preceded by a local ventricular electrogram at a cycle length of $300 \mathrm{msec}$. The QRS configuration was constant regardless of the pacing cycle lengths (Figure 2B). These findings suggested that AV conduction was solely via the anterior AV node and that the posterior His bundle was activated retrogradely through the anterior AV node in a decremental manner, at least at a short atrial pacing cycle length.

During the SVT, the earliest activation site was the posterior HBE (Figure $2 \mathrm{C}$ ). Considering that the nonfunctioning posterior AV node was the focus of this SVT, we applied radiofrequency current to the posterior HBE. Radiofrequency current application terminated the SVT immediately (Figure 3), and atrial burst stimuli could no longer induce the SVT after the application was continued for 120 seconds.

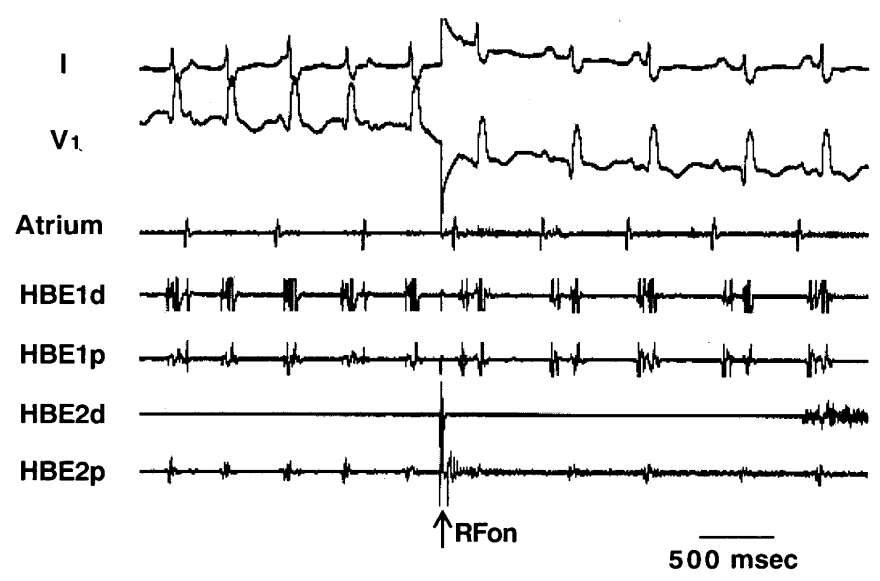

Figure 3. Body surface ECG leads I and V1, and intracardiac electrograms recorded in the atrium, the distal and proximal electrodes of mapping catheters which were located in HBE1 and HBE2 (HBE1d and HBE1p, and HBE2d and HBE2p, respectively) during the application of radiofrequency current. The current was applied to the site of HBE2d. The SVT and atrial cycle length were 380 and $540 \mathrm{msec}$, respectively, and VA dissociation with partial atrial capture was observed during the SVT. 


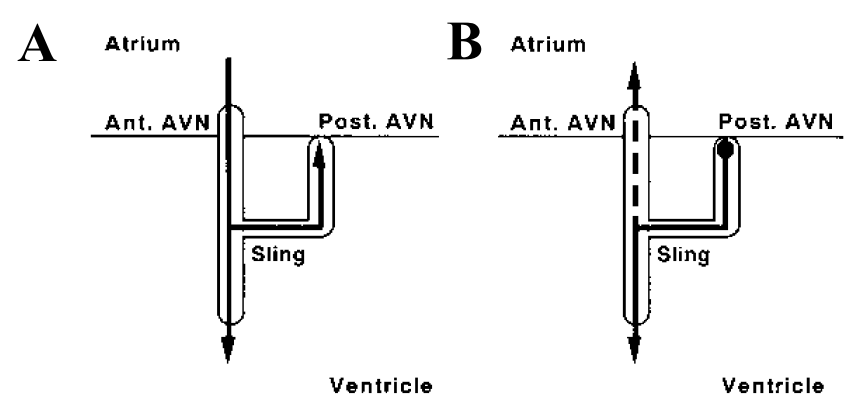

\begin{abstract}
Figure 4. Possible scheme of the cardiac conduction system in this case. Presumed activation sequences during atrial pacing and during the SVT are shown in panels A and B, respectively. A: The impulse from the atrium conducts to the ventricle via the anterior AV node. The posterior AV node is activated retrogradely through the anterior AV node and a sling. B: The SVT originates from the posterior $\mathrm{AV}$ node. The impulse conducts to the anterior AV node, and to the ventricle through a sling. The retrograde conduction via the anterior AV node is intermittent (broken line). Ant, Post, and AVN indicate anterior, posterior, and AV node, respectively.
\end{abstract}

\title{
DISCUSSION
}

The presence of dual AV nodes and a sling between them has been described in several congenital complex heart diseases. They include right isomerism, left isomerism with the morphologically right ventricle to the left of the morphologically left ventricle (1-loop), or with single ventricle of right ventricular type/indeterminate morphology, ${ }^{1)}$ situs inversus and AV concordance with double-outlet right ventricle, ${ }^{7)}$ and corrected transposition of the great arteries. ${ }^{2,3)}$ Although our patient had single atrium, he had polysplenia syndrome, which suggested that his cardiac malformation was embryologically equivalent to left atrial isomerism. Furthermore, he also had single ventricle of the right ventricular type (with rudimentary left ventricle). For these reasons, we anticipated the presence of dual AV nodes, which were identified by mapping along the common AV valve annulus. Although AV node to AV node reentrant tachycardia has been often observed in this setting, ${ }^{4-6)}$ the SVT in our patient was maintained even when VA block occurred. Because the distance between two AV nodes was considerably long (Figure 2A), the reentry circuit of the inter-AV node reentry should not be completed without the atrium. Accordingly, this type of tachycardia was unlikely in our patient.

In general, SVT with VA block suggests automatic junctional tachycardia, AV nodal reentrant tachycardia with an upper common pathway, intra-Hisian reentrant tachycardia, or AV reentrant tachycardia using concealed nodofascicu- 
lar bypass tract as a retrograde limb. ${ }^{8}$ The reproducible induction and termination of the SVT by atrial burst stimuli were unusual in automatic junctional tachycardia. Moreover, the failure to reset the SVT by ventricular extrastimulation did not favor AV reentrant tachycardia using the nodofascicular bypass tract, and the lack of intra-Hisian conduction delay was not characteristic of intra-Hisian reentrant tachycardia. In addition, the radiofrequency current application to the posterior AV nodal region actually eliminated the SVT. Consequently, AV nodal reentrant tachycardia with an upper common pathway confined to the posterior AV nodal region was a possible mechanism of this SVT, although direct evidence supporting the diagnosis of AV nodal reentrant tachycardia could not be obtained and junctional tachycardia due to micro-reentry or triggered activity might be the mechanism.

Figure 4 shows the possible scheme of the cardiac conduction system in the present case. The finding in Figure 2B suggested that the anterior AV node was responsible for $\mathrm{AV}$ conduction and that the posterior $\mathrm{AV}$ node was the bystander. However, it remains unknown whether the conduction between the atrium and the posterior $\mathrm{AV}$ node was really absent during atrial pacing at a long cycle length or during the SVT. Figures $2 \mathrm{~B}$ and $2 \mathrm{C}$ show that the $\mathrm{HV}$ interval recorded at the anterior AV node was $55 \mathrm{msec}$ during atrial pacing and $0 \mathrm{msec}$ during the SVT. This observation supported the view that two AV nodes were linked not through the ventricular muscle but possibly through a sling at the infra-Hisian level.

In summary, we have described SVT originating from the nonfunctioning posterior AV node in the univentricular heart with single atrium and polysplenia syndrome. It is believed that the anterior and posterior AV nodes were connected to each other via a sling at the infra-Hisian level. This SVT was assumed to be AV nodal reentrant tachycardia confined to the posterior AV nodal region, and was successfully ablated by applying radiofrequency current to the posterior AV node.

\section{REFERENCES}

1. Dickinson DF, Wilkinson JL, Anderson KR, Smith A, Ho SY, Anderson RH. The cardiac conduction system in situs ambiguus. Circulation 1979; 59: 879-85.

2. Anderson RH, Becker AE, Arnold R, Wilkinson JL. The conducting tissues in congenitally corrected transposition. Circulation 1974; 50: 911-23.

3. Wilkinson JL, Smith A, Lincoln C, Anderson RH. Conducting tissues in congenitally corrected transposition with situs inversus. Br Heart J 1978; 40: 41-8.

4. Saul JP, Walsh EP, Triedman JK. Mechanisms and therapy of complex arrhythmias in pediatric patients. J Cardiovasc Electrophysiol 1995; 6: 1129-48. (Review)

5. Wu MH, Wang JK, Lin JL, et al. Supraventricular tachycardia in patients with right atrial isomerism. J Am Coll Cardiol 1998; 32: 773-9. 
6. Epstein MR, Saul JP, Weindling SN, Triedman JK, Walsh EP. Atrioventricular reciprocating tachycardia involving twin atrioventricular nodes in patients with complex congenital heart disease. J Cardiovasc Electrophysiol 2001; 12: 671-9.

7. Anderson RH, Thapar MK, Arnold R, Jones RS. Study of conducting tissue in a case of ventricular pre-excitation. Br Heart J 1973; 35: 566-9.

8. Hamdan MH, Kalman JM, Lesh MD, et al. Narrow complex tachycardia with VA block: diagnostic and therapeutic implications. Pacing Clin Electrophysial 1998; 21: 1196-206. 